\title{
Sensitivity Analysis of the DARHT-II 2.5MV/2kA Diode
}

\author{
Enrique Henestroza
}

December 22, 2006 


\section{Introduction}

This report summarizes the study of the tolerance limits on the assembly of the cathode and the Pierce electrode for the DARHT-II diode (2.5 MV, $2 \mathrm{kA}$ case), performed through a series of computer simulations using the PIC code WARP [1]. We have considered sources of beam quality degradation like the errors in axial and transverse positioning, and the size of the radial gap between the cathode and the Pierce electrode (shroud). The figure of merit was chosen to be the RMS beam (edge) emittance at a distance of 1 meter from the cathode, as defined by

$$
\varepsilon_{x}=4 \beta \gamma \sqrt{\left\langle x^{2}\right\rangle\left\langle x^{\prime 2}\right\rangle-\left\langle x x^{\prime}\right\rangle^{2}} .
$$

The analysis shows that to position the cathode at the correct axial and transverse location is more important than the size of the radial gap.

\section{Diode Geometry}

The diode geometry is described in Figure 1.

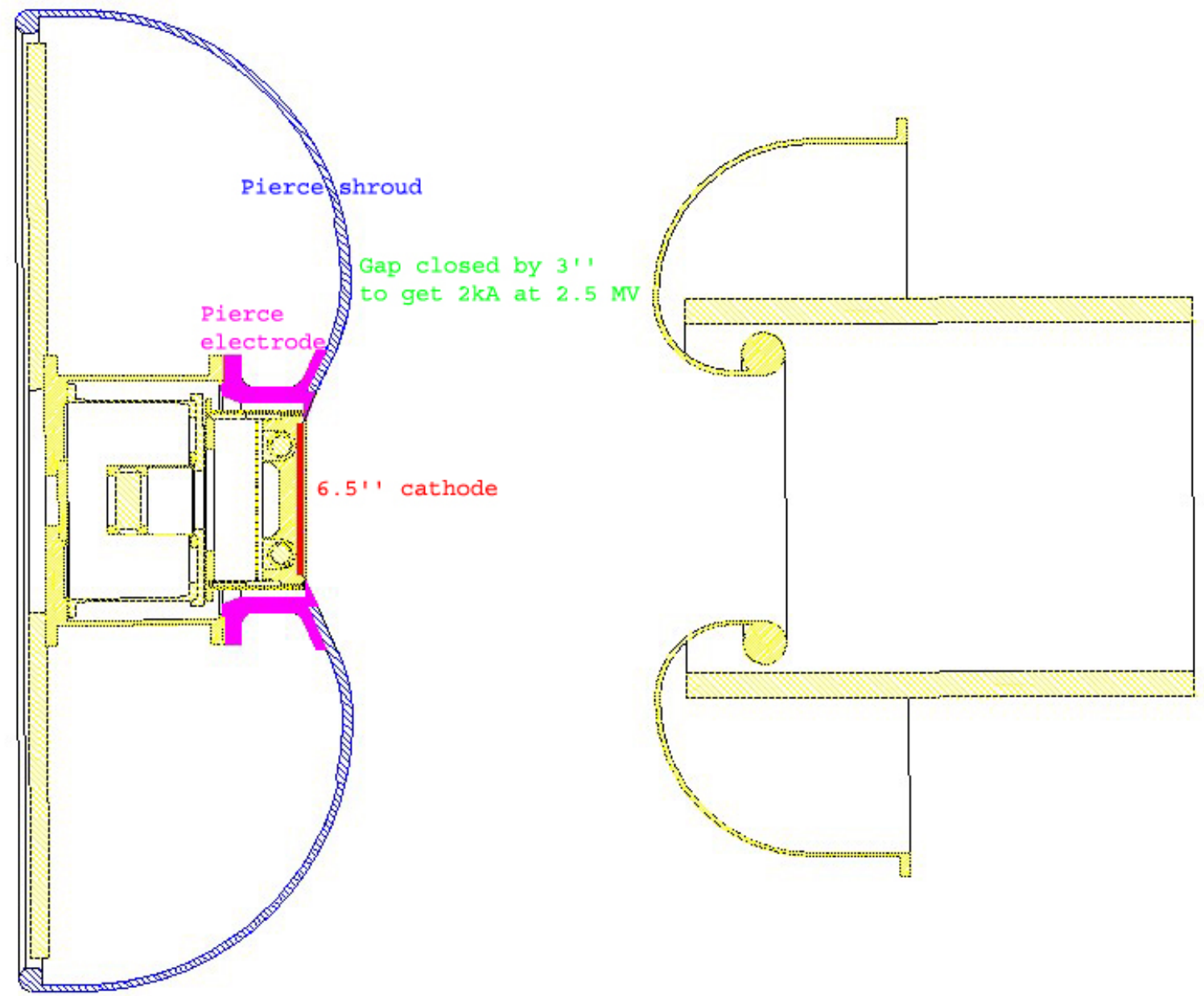

Figure 1 Schematic of diode showing relevant components. 


\subsection{Electrostatic fields}

The fields for the proposed 2.5 MV, $2 \mathrm{kA}$ diode are essentially the same as in the original $3.2 \mathrm{MV}, 2 \mathrm{kA}$ diode, i.e., peak fields of $140 \mathrm{kV} / \mathrm{cm}$ on the anode and $120 \mathrm{kV} / \mathrm{cm}$ on the cathode as shown in Figure 2 as calculated from EGUN [2].
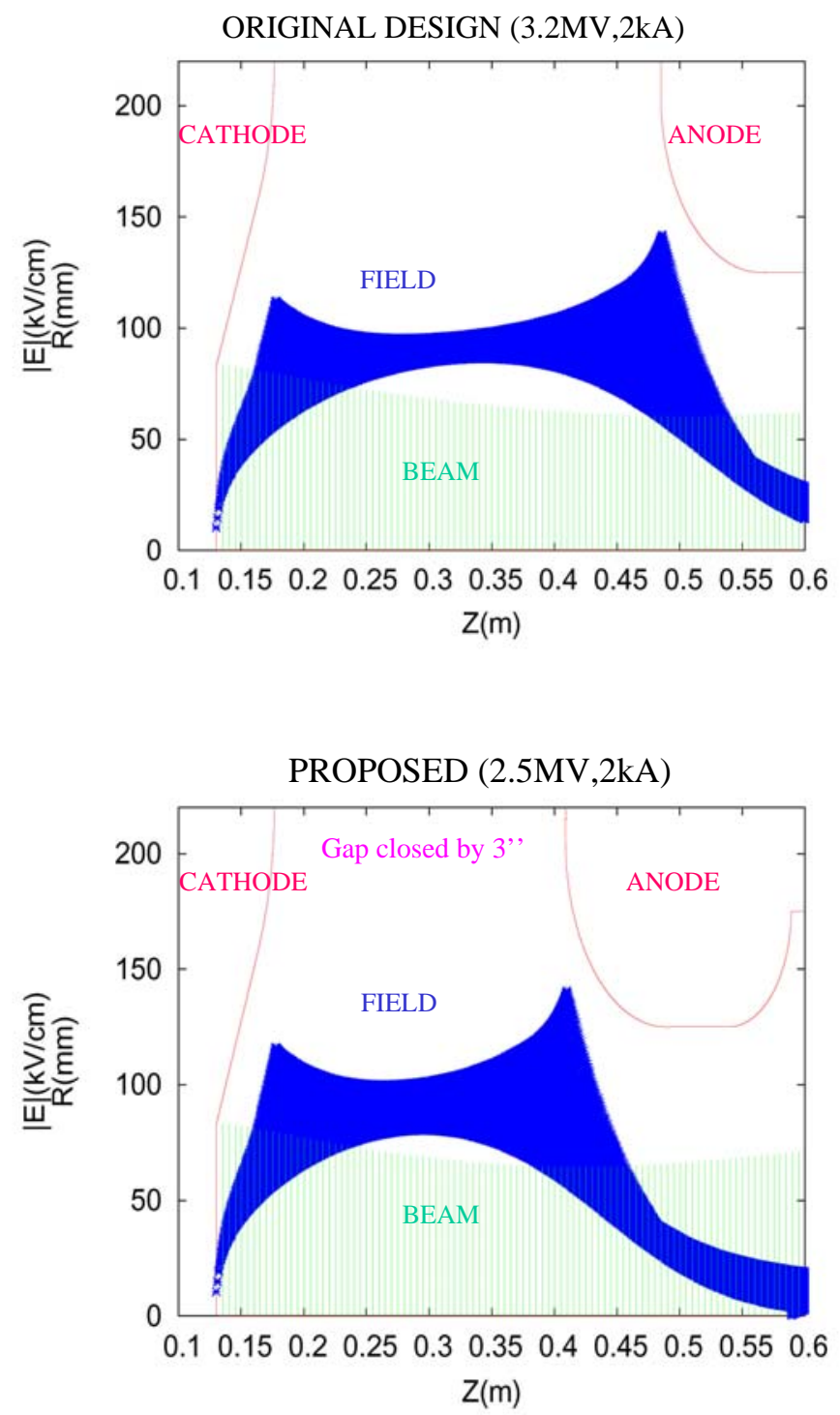

Figure 2 EGUN simulation of (top) original and (bottom) proposed diode geometries, showing (green) the beam envelope, and (blue) the field along the axis plotted for all radii. 


\section{Diode assembly errors}

\subsection{Introduction}

The effect on the beam quality due to errors in axial positioning and the size of the radial gap between the cathode and the Pierce electrode were considered during the initial design of the DART-II diode. Figure 3 shows the effect on the beam dynamics due to a radial gap, and a protrusion or recession of the cathode relative to the Pierce electrode.
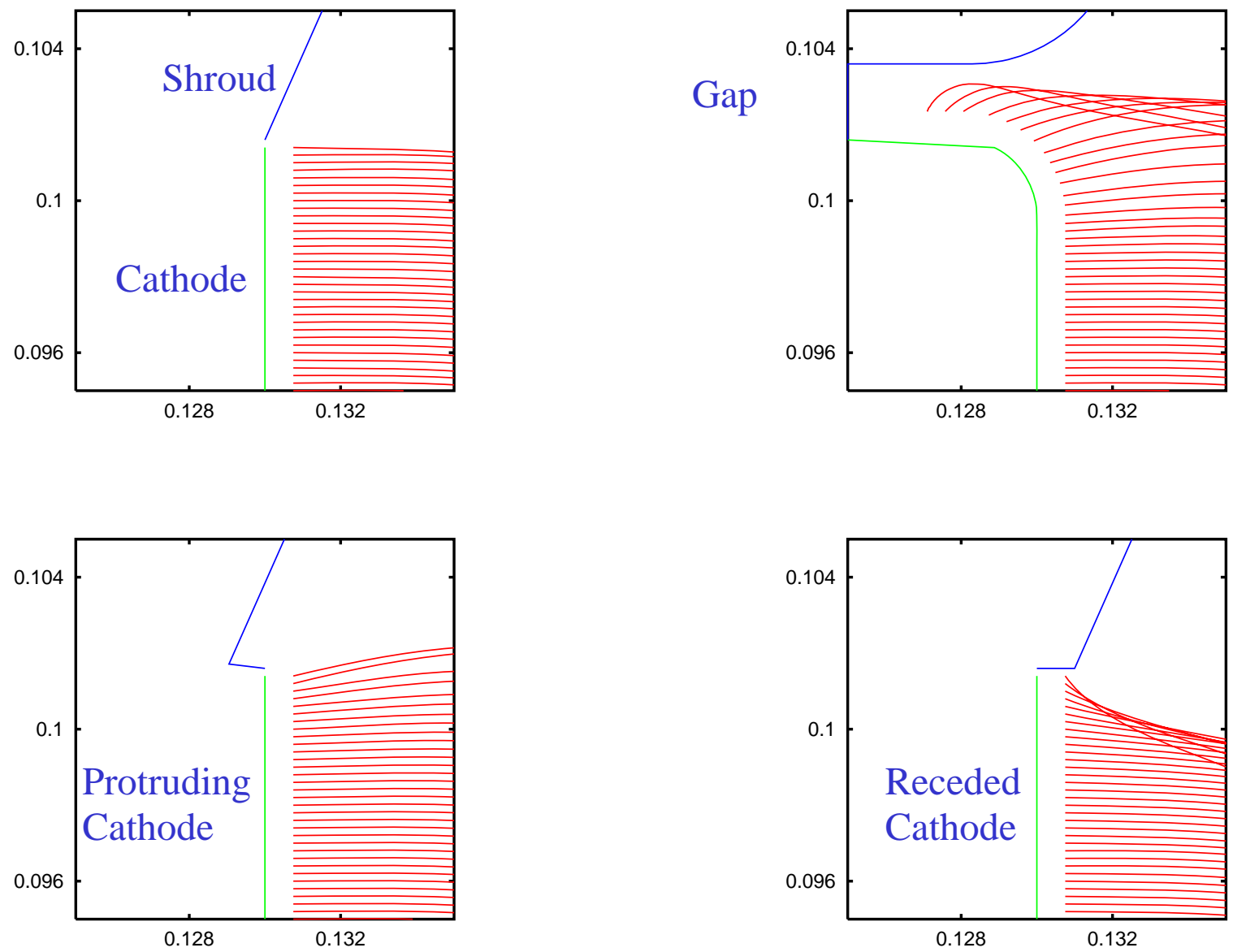

Figure 3 EGUN calculations for several cathode-shroud assembly errors.

The figure of merit was chosen to be the beam (edge) emittance. The analysis showed that it is more important to position the cathode at the correct axial location than the size of the radial gap. Figure 4 shows the effect on the emittance due to the assembly errors considered. 


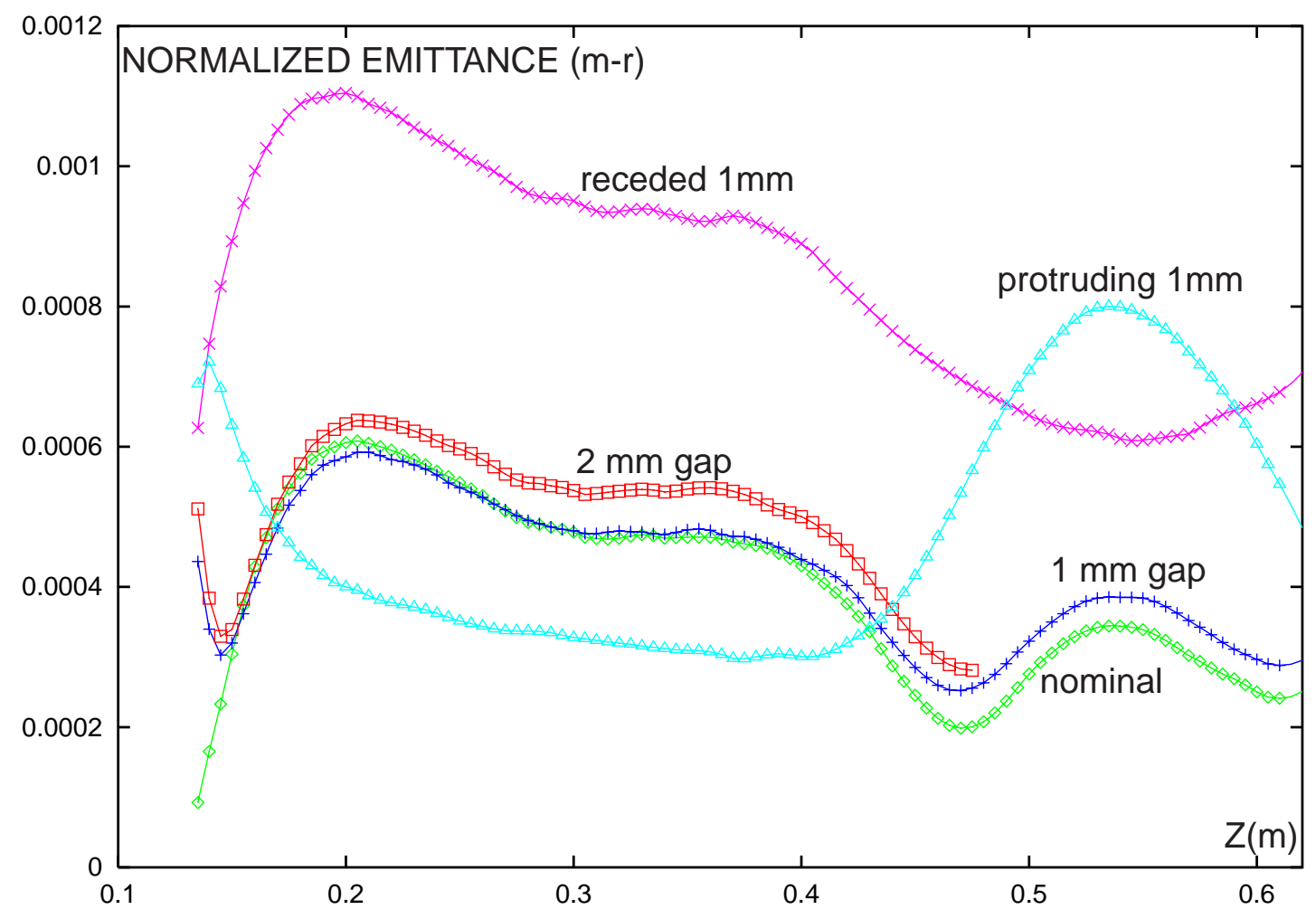

Figure 4 EGUN calculations showing the emittance along diode axis for several cathode-shroud assembly errors.

The model used to obtain the original results (EGUN simulations) did not include emission from the radial surface being exposed by the radial gap or the protrusion. A new set of calculations using the particle-in-cell code WARP, that are the subject of this report, include emission from radial surfaces (and sharp corners), as well as higher resolutions obtained by using mesh refinement methods.

One has to keep in mind that a change in the geometry of the diode changes the amount, and distribution, of the current emitted, as well as the beam dynamics as the beam propagates down the beam line. As an example, Figure 5 shows the change in emittance and envelope profile that occurs when the cathode is receded 80 mils with respect to the nominal shroud location which produces a current drop of 100 Amperes

We define the nominal location of the cathode relative to the shroud, for the case of no radial gap, when the edge of the cathode and shroud coincide. 


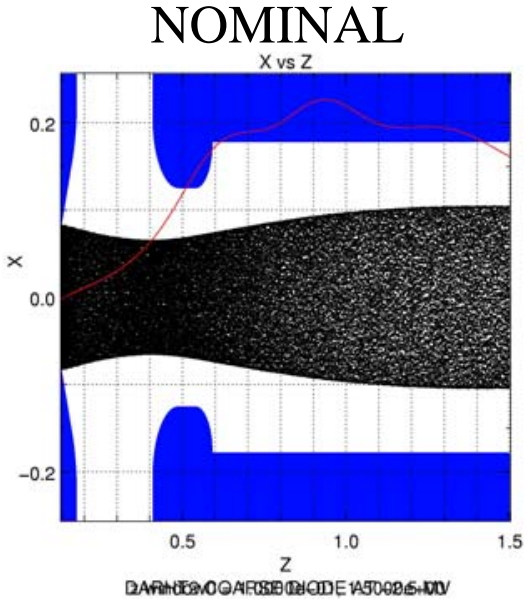

Step $650, T=0.0065 \mathrm{e}-6 \mathrm{~s}$, Zbeam $=0.0000 \mathrm{~m}$ DARHT2 PIC RZ SIMULATIONS

Enrique Henestroza warp $\mathrm{r} 2$

DARHT2INJ2.5MV

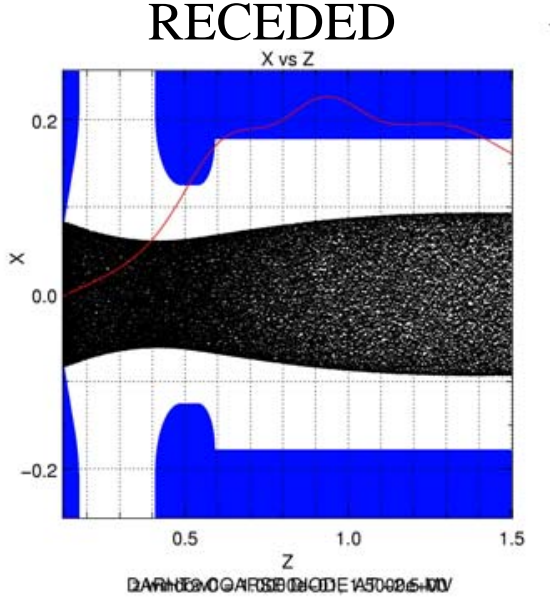

Step $650, T=0.0065 \mathrm{e}-6 \mathrm{~s}$, Zbeam $=0.0000 \mathrm{~m}$ DARHT2 PIC RZ SIMULATIONS

Enrique Henestroza warp 12

DARHT2/NJ2.5MV 2KAREC80milsp

\section{NOMINAL}

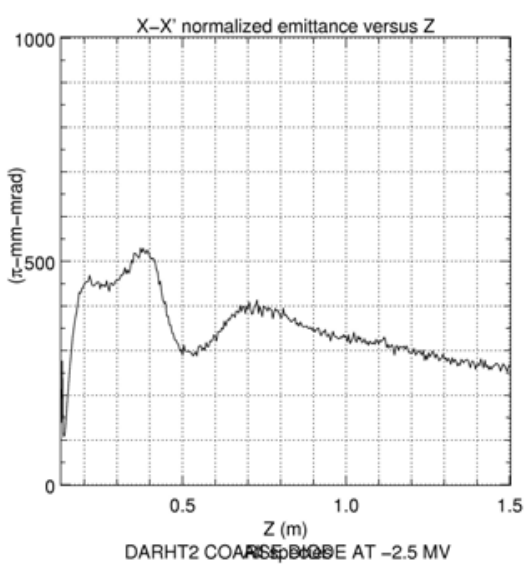

Step $650, T=0.0065 \mathrm{e}-6 \mathrm{~s}$, Zbeam $=0.0000 \mathrm{~m}$

DARHT2 PIC RZ SIMULATIONS

Enrique Henestroza warp r2

DARHT2 INU2.5MV

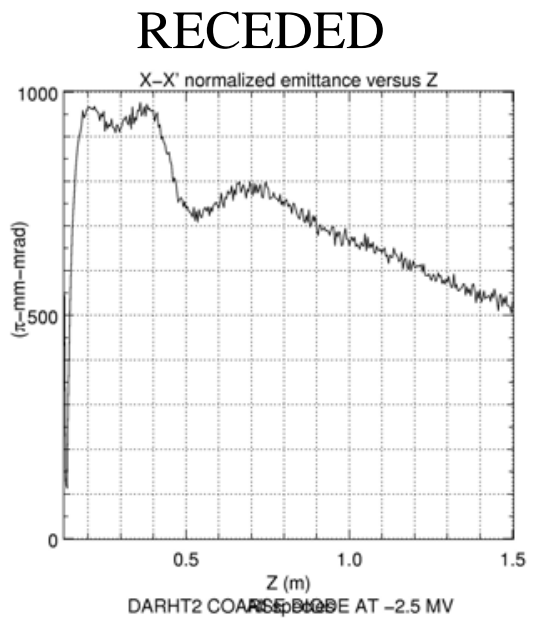

Step $650, T=0.0065 e-6 \mathrm{~s}$, Zbeam $=0.0000 \mathrm{~m}$

DARHT2 PIC RZ SIMULATIONS

Enrique Henestroza warp ra

DARHT2 2N 2.5MV 2KAREC80milsp

Figure 5 WARP calculations showing (top) the beam envelope and (bottom) emittance along diode axis for a cathode-receded 80 mils from the nominal shroud location. 


\subsection{Nominal diode geometry and FOM}

Since we want to study the effect of misalignments on the quality of the extracted beam, one has to define a baseline geometry that will be used to quantify the effect. In the case of a two-dimensional diode (slab geometry) and no aperture (closed system), it is well known that the Pierce solution geometry generates a laminar beam with zero emittance; but in relativistic, axially symmetric systems with a big aperture, there are no (known) solution that generates a laminar beam with zero emittance; therefore there is no "ideal" solution that we can take as reference. The best we can do is to design a diode that extracts a beam that meets the requirements of current, voltage, envelope size and emittance at certain point in the beam line, and design the downstream section in such a way as to maintain the beam quality within prescribed limits For the DARHT-II diode, we take as the reference, the nominal diode geometry as described in Figures 1 and 6 .

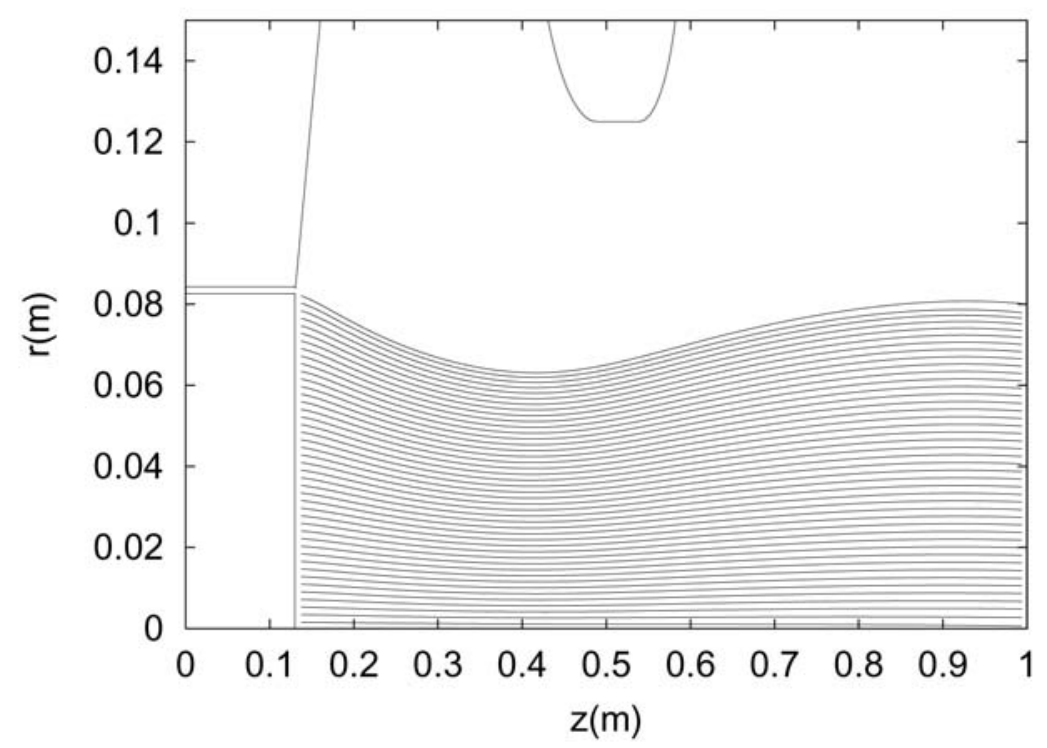

Figure 6 EGUN simulation of the nominal diode configuration.

As figure of merit (FOM) to compare different geometries we have chosen the RMS beam (edge) emittance at a distance of 1 meter from the cathode, where the lower the emittance the better the beam quality. Although the RMS emittance is a good parameter to use as FOM, it may happen that another configuration with a higher emittance at the given location may generate a better beam quality when propagated for a longer distance downstream. We will find this kind of situation with geometry configurations where the cathode is protruding.

In general, the density distribution in the radial direction of the beam has to be as uniform as possible. In particular beam components whose behavior departs from the general trend of the beam should be avoided; these components are known as "translaminar" 
components, and they are generated by errors from an otherwise nominal geometry. Figures 7 and 8 show translaminar components generated by a receding and protruding cathode respectively.

The FOM should be qualified therefore by minimizing the amount of translaminar current.

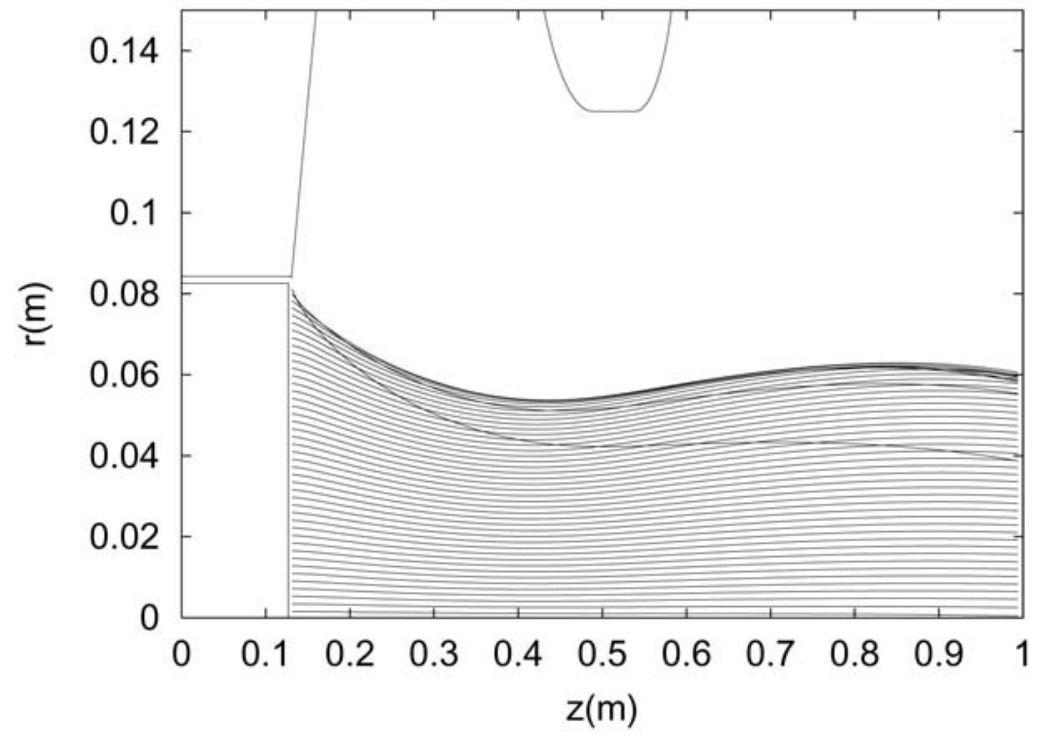

Figure 7 Translaminar beam components generated by a receding cathode.

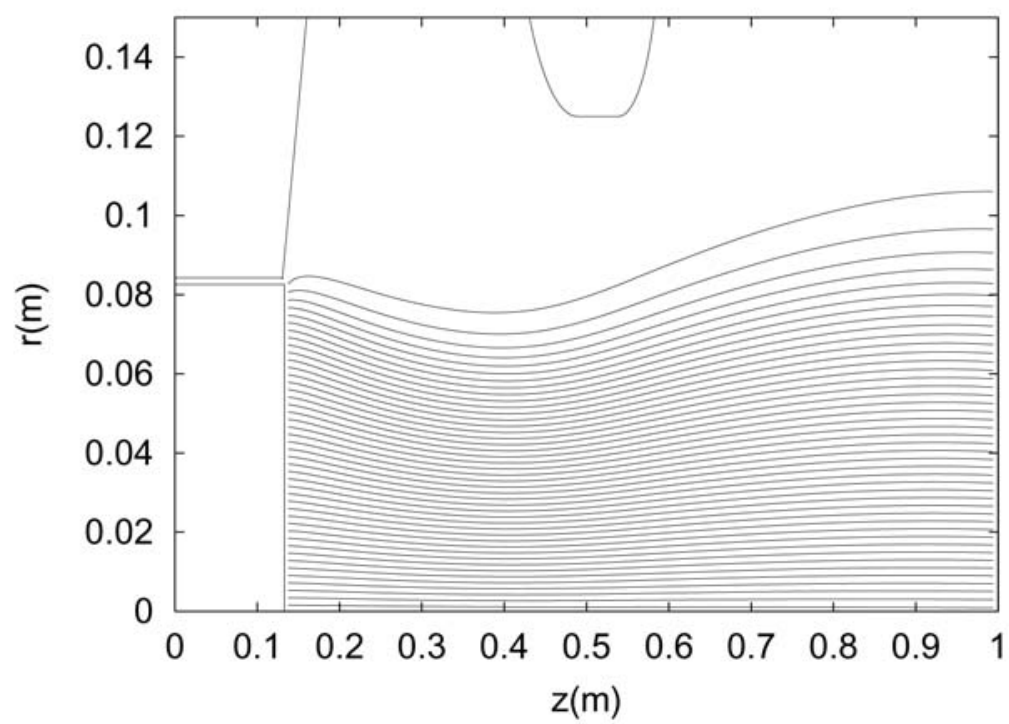

Figure 8 Translaminar beam components generated by a protruding cathode. 


\subsection{Cathode receding/protruding relative to nominal location with respect to shroud}

The nominal location of the cathode relative to the shroud, for the case of no radial gap, is when the edge of the cathode and shroud coincide. Due to assembly errors and heat expansion effects, the cathode may end up either receding or protruding relative to the nominal position by \pm 20 mils. Figures 9 (a,b,c) show the emittance, the current and the radial envelope along the diode axis for several cathode-shroud distances from the nominal location.

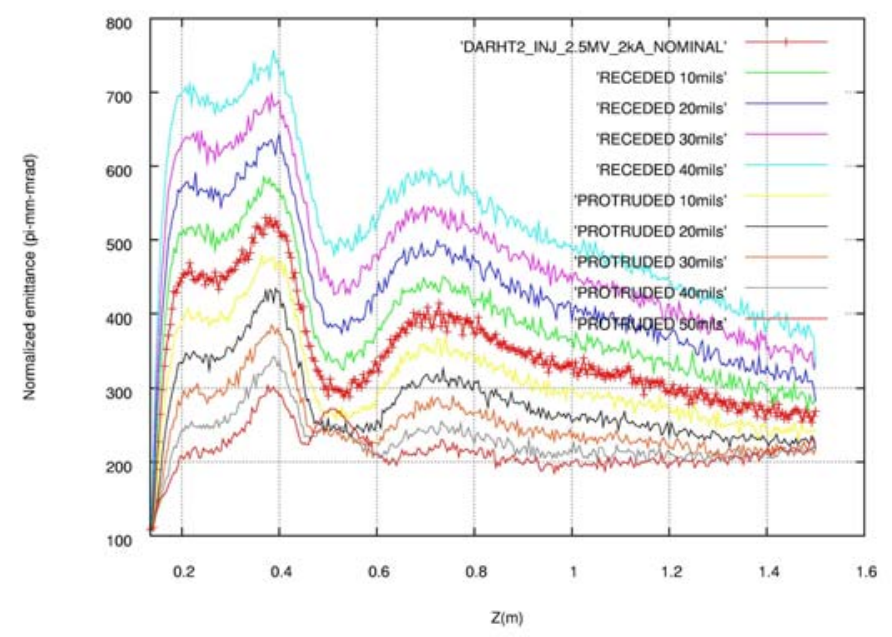

Figure 9a WARP calculated emittance along the diode axis for a cathode-receding or protruding from the nominal shroud location.

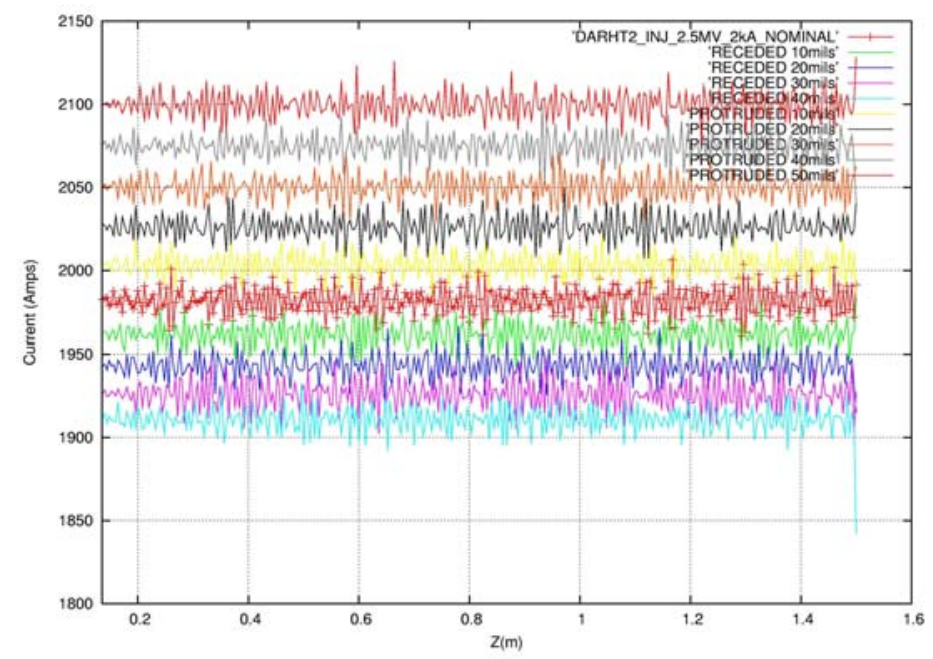

Figure 9b WARP calculated current along the diode axis for a cathode-receding or protruding from the nominal shroud location. 


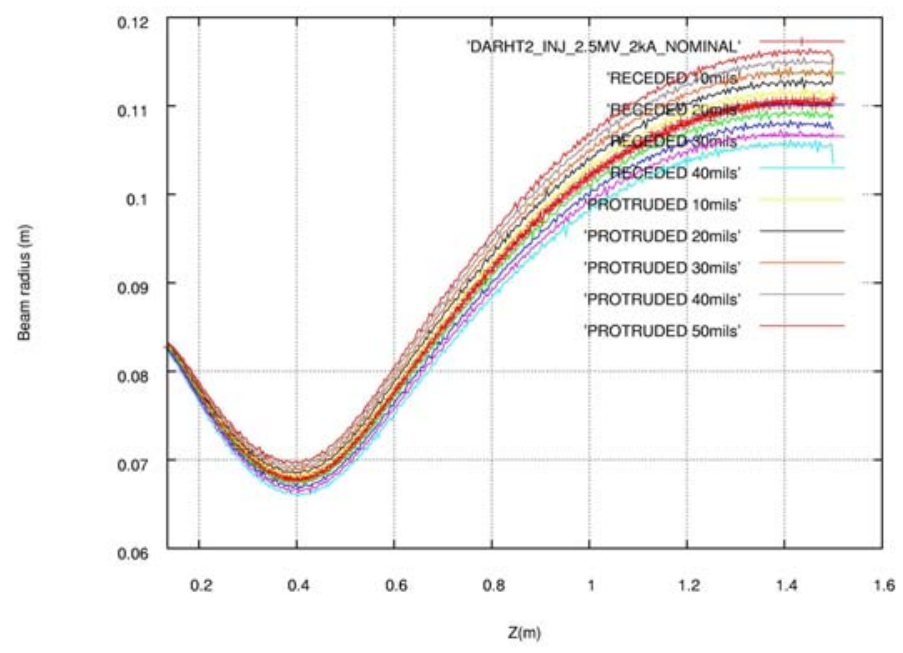

Figure 9c WARP calculated envelope along the diode axis for a cathode-receding or protruding from the nominal shroud location.

As was discussed in Section 3.2, the fact that the emittance for the cases of protruding cathode is consistently lower than for the baseline case does not imply that these configurations will generate a better beam quality when propagated for a longer distance downstream. Protruding (Figure 8) or receding (Figure 7) cathode configurations generate translaminar components which are not matched to follow the envelope of the main body of the beam and therefore will give rise to higher emittance values at some point downstream. This is apparent in Figure 4 where the protruding case goes from lower to higher emittance than the nominal case as the beam propagates downstream.

In fact the amount of translaminar current generated by the non-nominal geometry is less for the receding than for the protruding cases. A 40 mil receding geometry generates 1.1 Amperes and a 40 mils protruding geometry generates 6.6 Amperes of translaminar current.

We could then have as the most reasonable conclusion from the set of WARP runs presented in Figure 9 (a,b,c), that an error in the axial position of the cathode relative to the Pierce electrode of say, \pm 20 mils will increase the normalized (edge) emittance of the beam by about $50 \pi$-mm-mr (out of $250 \pi$-mm-mr), increase (protruding) or decrease (receding) the current by about 50 Amperes, and increase (protruding) or decrease (receding) the beam size by about $2 \mathrm{~mm}$. 


\subsection{Radial gap between cathode and shroud}

In order to prevent the hot cathode to lose heat to the Pierce electrode, there is a need to provide a gap between them. A relatively large gap of $\sim 60$ mils in cold conditions, and $\sim 40$ mils in hot conditions, should provide enough clearance to make sure that the cathode does not touch the Pierce electrode, when we take into account assembly errors and uncertainties in heat expansion effects.

The nominal location of the cathode relative to the shroud, for the case when there is need to have a radial gap, is obtained by starting from the nominal geometry (see Section 3.2) and removing material from the Pierce electrode to generate the gap without changing its location or shape.

Figures 10 (a,b,c) show the emittance, the current and the radial envelope along the diode axis for several cathode-shroud radial gaps.

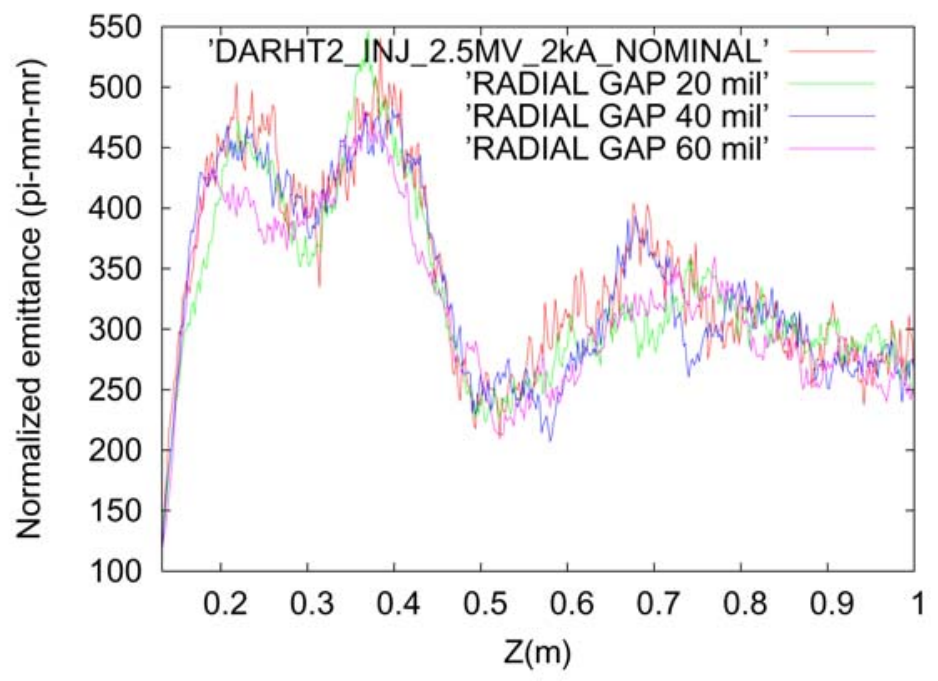

Figure 10a WARP calculated emittance along the diode axis for several cathode-shroud radial gaps. 


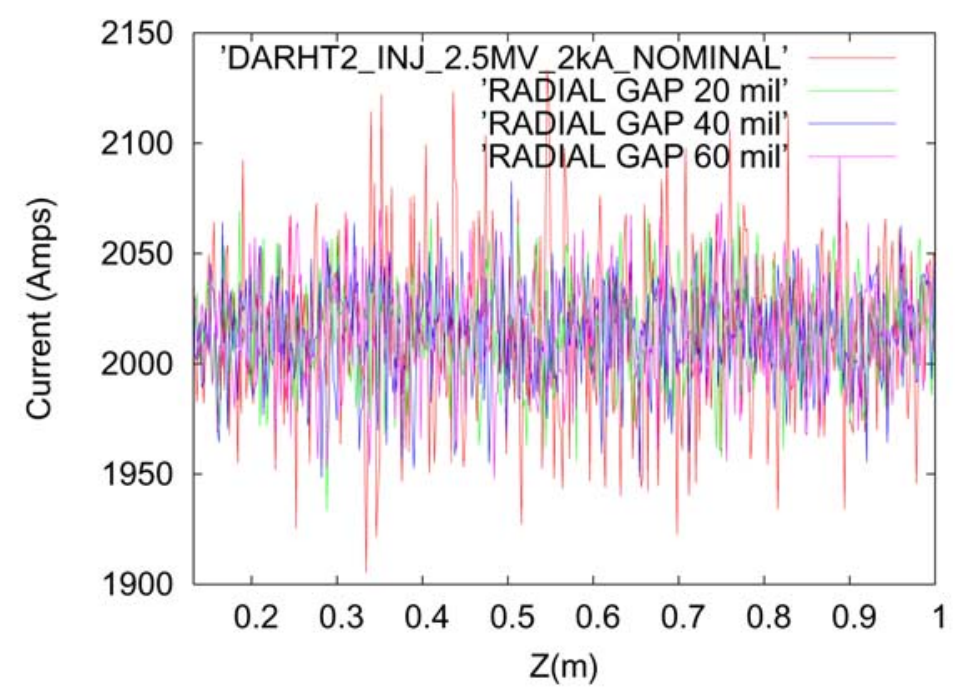

Figure 10b WARP calculated current along the diode axis for several cathode-shroud radial gaps.

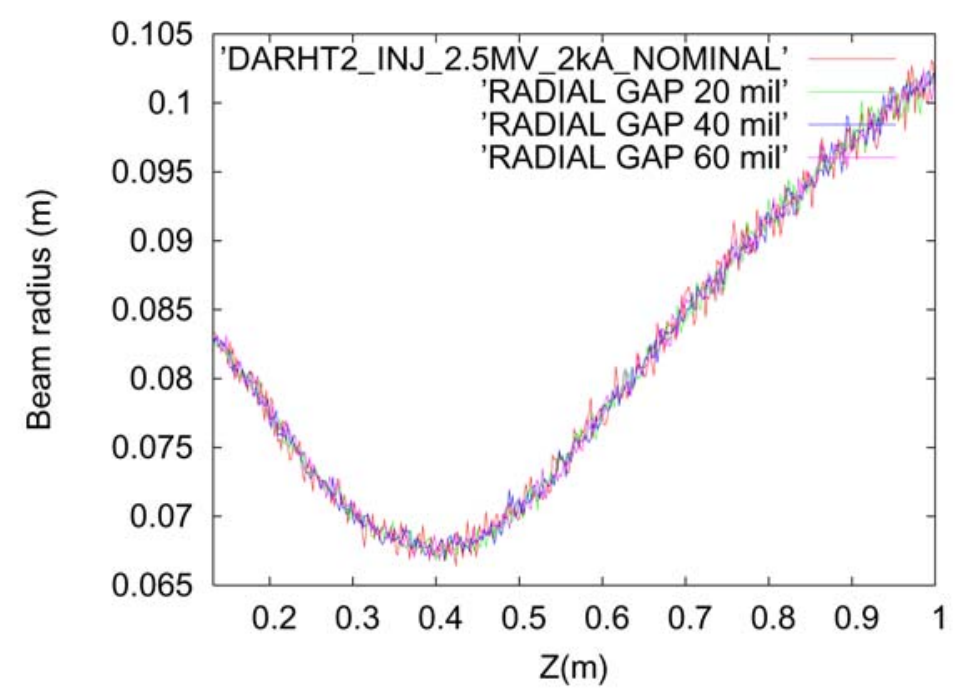

Figure 10c WARP calculated envelope along the diode axis for several cathode-shroud radial gaps.

It is evident from Figures 10 (a,b,c) that in the case of a small radial gap, the beam parameters are virtually unchanged. The amount of translaminar current increases from 1.1 Amperes for a 20 mil gap, to 3.2 Amperes for a 40 mil gap, and up to 5.3 Amperes for a 60 mil gap. 


\subsection{Cathode transverse offset}

The relatively large gap of $\sim 60$ mils in cold conditions, that provides enough clearance to make sure that the cathode does not touch the Pierce electrode, also introduces the risk of further errors in the transverse positioning of the cathode, which will break the rotational symmetry of the assembly.

WARP calculations in three dimensions are computationally quite expensive to perform; furthermore, some beam parameters like the emittance are quite sensitive to the computational mesh used. This makes a one-to-one comparison between the RZ calculations and the XYZ calculations very difficult to make. Therefore, we have chosen to show the relative beam quality degradation by comparing only XYZ calculations.

Figures $11(\mathrm{a}, \mathrm{b})$ show the transverse emittance along the diode axis for two XYZ WARP calculations. Since it is almost impossible, within our computational resources (even with mesh refinement), to model smaller gaps, we have chosen to model a gap of 200 mils and an offset of 100 mils.

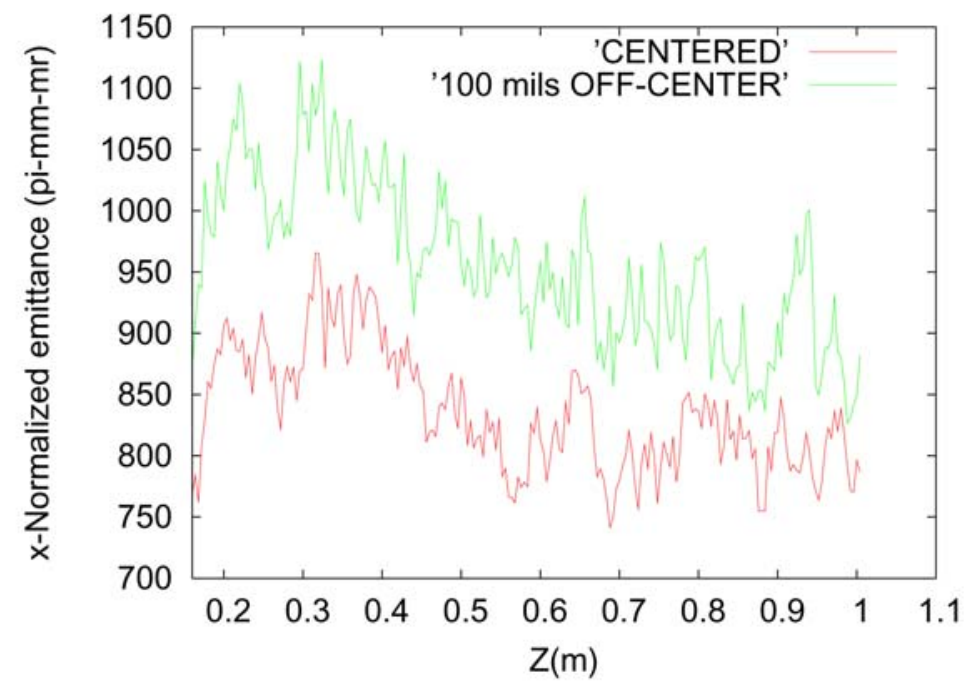

Figure 11a WARP calculated horizontal emittance along the diode axis for zero and 100 mil horizontal offset. 


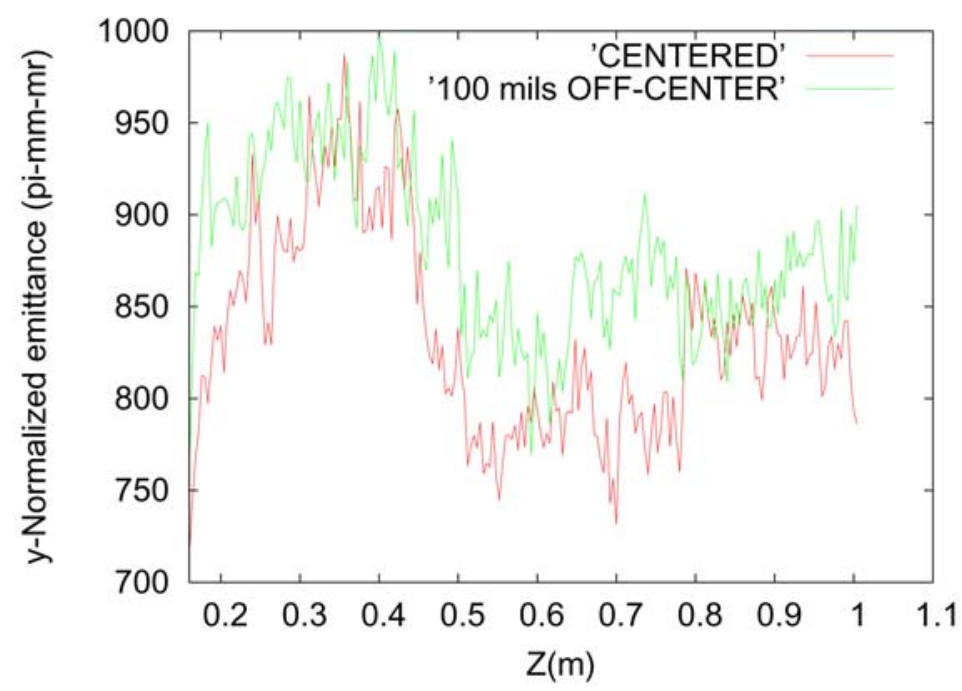

Figure 11b WARP calculated vertical emittance along the diode axis for zero and 100 mil horizontal offset.

Except for the emittance, all other beam parameters are virtually unchanged. The centroid motion is damped by the external solenoidal magnetic field. From this calculation we expect an increase of about $20 \%$ in emittance in the direction of the offset when we have an offset of half the radial gap.

\section{Conclusion}

In order to quantify the relationship between the amount of translaminar current and the increase in emittance, additional analysis is required; the beam should be followed to the end of the machine, and the beam degradation quantified. Since this type of simulation cannot be done in a single self consistent calculation, a mixture of 3D, RZ and XY-slice calculations must be used

Heat analysis of the diode system has shown [3] that locating the cathode receded by 20 mils and allowing a 60 mils radial gap in cold conditions, will end up with a geometry in hot conditions where the cathode is located at the nominal axial location and the radial gap is reduced to 40 mils, which will degrade the beam quality by a small amount (negligible emittance increase and the emission of 3.2 Amperes of translaminar current). The radial gap should provide enough clearance to ensure that the cathode does not touch the Pierce electrode. Also a 20 mils transverse position tolerance (half the radial gap in hot conditions) should limit the beam degradation to about $20 \%$ increase in emittance.

In conclusion, the recommended cathode position in hot conditions is the nominal axial location, the radial gap should be less than 40 mils and the radial offset less than 20 mils (half the radial gap). The tolerance should be within 4 mils. 


\section{References}

1. D. P. Grote, A. Friedman, I. Haber, and S. S. Yu, Fusion Eng. Design 32-33, 133 (1996).

2. W. B. Herrmannsfeldt, "EGUN - An Electron Optics and Gun Design Program," SLAC-Report 331 (1988).

3. M. Leitner, Private communication (December 18, 2006). 